\title{
Modeling Nonlinear Memory Effects on the AM/AM, AM/PM and Two-Tone IMD in Microwave PA Circuits
}

\author{
Pedro M. Cabral, José C. Pedro, Nuno B. Carvalho \\ Instituto de Telecomunicações, Universidade de Aveiro, Campus Universitário de Santiago, \\ 3810-193 Aveiro, Portugal
}

Received 14 March 2005; accepted 20 August 2005

\begin{abstract}
This article deals with the nonlinear memory effects observed on both AM/AM and $\mathrm{AM} / \mathrm{PM}$ conversions and swept frequency-separation two-tone intermodulation-distortion (IMD) tests made on microwave power-amplifier (PA) circuits. This study is theoretically introduced via an analytic Volterra series analysis, then complemented with large-signal harmonic balance and envelope simulations of a GaN HEMT-based microwave PA built with different load terminations. (c) 2005 Wiley Periodicals, Inc. Int J RF and Microwave CAE 16: 13-23, 2006.
\end{abstract}

Keywords: AM/AM; AM/PM; harmonic terminations; memory effects; two-tone IMD

\section{INTRODUCTION}

Nowadays, emerging terrestrial and space communication systems require complex modulation schemes with high spectral efficiency. Almost all of those systems have at least one microwave power amplifier (PA), which is usually the last active block in any transmitter system, handling the highest levels of RF signal and supply power. Operating these PAs near saturation increases signal distortion but improves efficiency, justifying the effort spent on an accurate PA nonlinear characterization to optimize that tradeoff. In order to estimate the impact that the power amplification has in narrowband communication systems, the nonlinear behaviour of a PA is usually described using the AM/AM and AM/PM conversions [1].

The above mentioned conversions consist in the transformation by the nonlinear active device of the

Correspondence to: P. Cabral; email: pcabral@av.it.pt.

DOI $10.1002 /$ mmce.20127

Published online 14 November 2005 in Wiley InterScience (www.interscience.wiley.com). input amplitude variations, that is, amplitude modulation (AM), into variations of the output amplitude or phase, that is, amplitude modulation (AM) or phase modulation (PM), respectively.

$\mathrm{AM} / \mathrm{AM}$ is particularly important in systems based on amplitude modulation; while AM/PM has its major impact in nonconstant envelope phase-modulation formats.

Figure 1 shows a 64-QAM constellation diagram, in which it is possible to see the amplitude and phase conversions' impact on the symbol decoding.

$\mathrm{AM} / \mathrm{AM}$ and $\mathrm{AM} / \mathrm{PM}$ conversions are usually measured statically using a vector network analyzer (VNA). In communication signals, the envelope carries the information as amplitude and phase modulation. Thus, $\mathrm{AM} / \mathrm{AM}$ and $\mathrm{AM} / \mathrm{PM}$ conversions are essential signal-transmission impairments that have to be accurately characterized [2].

This article is devoted to providing a comprehensive study of the PA's in-band and out-of-band output terminations' impact on the static and dynamic signaldistortion impairments: AM/AM and AM/PM conversions and swept frequency-separation two-tone inter- 


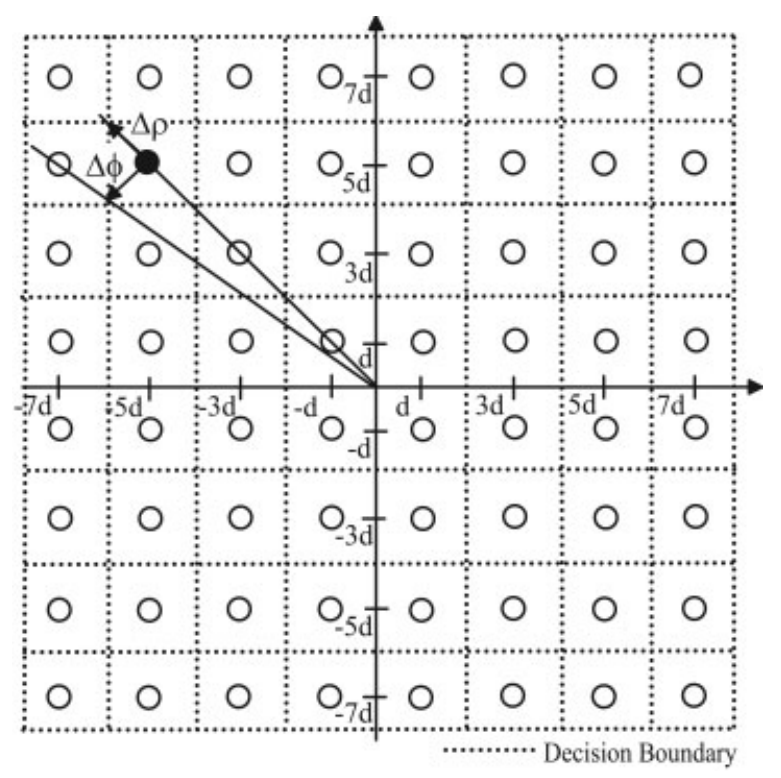

Figure 1. 64-QAM constellation diagram.

modulation-distortion (IMD) tests. For that, it is divided in five different sections. Section I introduces the work. Section II briefly presents the theoretical model formulation that supports all the following assumptions. Section III describes several envelope simulations of AM/AM and AM/PM done with different load terminations. Section IV complements this study with the memory-effects manifestation on the two-tone IMD tests. Finally, Section V concludes the work by summarizing its most important achievements.

\section{THEORETICAL MODEL FORMULATION}

Figure 2 presents a generic FET-based PA circuit used for the nonlinear analysis. As can be seen in the figure, $i_{D S}\left(v_{G S}, v_{D S}\right)$ is a nonlinear function dependent on two control voltages: $v_{G S}$ and $v_{D S}$. Using a loworder Taylor series expansion, we obtain

$$
\begin{array}{r}
i_{D S}\left(\nu_{G S}, \nu_{D S}\right)=I_{D S}+G_{m} \nu_{g s}+G_{d} \nu_{d s}+G_{m 2} \nu_{g s}^{2} \\
+G_{m d} \nu_{g s} \nu_{d s}+G_{d 2} \nu_{d s}^{2}+G_{m 3} \nu_{g s}^{3}+G_{m 2 d} \nu_{g s}^{2} \nu_{d s} \\
+G_{m d 2} \nu_{g s} \nu_{d s}^{2}+G_{d 3} \nu_{d s}^{3}
\end{array}
$$

Applying a mildly nonlinear Volterra series analysis to this circuit, where $v_{g s}(t)$ and $v_{d s}(t)$ are the input and output, respectively, we can obtain the first three Volterra frequency-domain nonlinear transfer functions (NLTFs): $H_{n}\left(\omega_{1}, \ldots, \omega_{n}\right)$ with $n=1 \ldots 3$, [3].

We define the auxiliary function $F_{C}(\omega)$ as

$$
F_{C}(\omega)=\frac{Z_{L}(\omega)}{1+G_{d s} \cdot Z_{L}(\omega)}
$$

The generic NLTFs are presented in the following equations:

$$
H_{1}(\omega)=-G_{m} \cdot F_{C}(\omega)
$$

$$
\begin{aligned}
H_{2}\left(\omega_{1}, \omega_{2}\right)= & -F_{C}\left(\omega_{1}+\omega_{2}\right) \cdot\left\{G_{m 2}+\frac{1}{2} G_{m d} \cdot\left[H_{1}\left(\omega_{1}\right)\right.\right. \\
& \left.\left.+H_{1}\left(\omega_{2}\right)\right]+G_{d 2} \cdot H_{1}\left(\omega_{1}\right) \cdot H_{1}\left(\omega_{2}\right)\right\}
\end{aligned}
$$

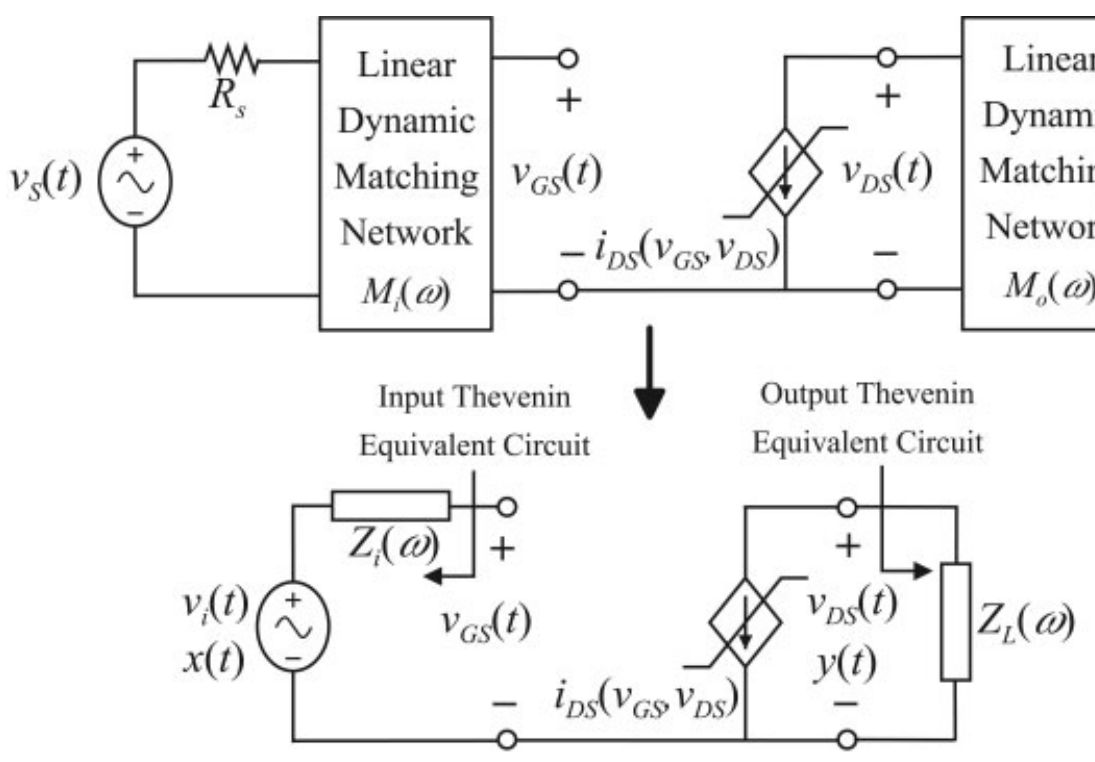

Figure 2. Simplified FET-based PA circuit used for the nonlinear analysis. 


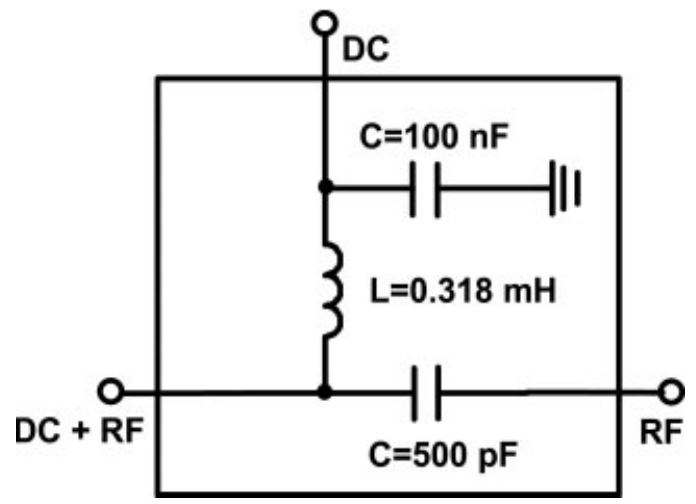

Figure 3. Nonideal bias-T.

$$
H_{3}\left(\omega_{1}, \omega_{2}, \omega_{3}\right)=-F_{C}\left(\omega_{1}+\omega_{2}+\omega_{3}\right)
$$

$$
\begin{gathered}
\cdot\left\{G_{m 3}+\frac{1}{3} G_{m 2 d} \cdot\left[H_{1}\left(\omega_{1}\right)+H_{1}\left(\omega_{2}\right)+H_{1}\left(\omega_{3}\right)\right]\right. \\
+\frac{1}{3} G_{m d 2} \cdot\left[H_{1}\left(\omega_{1}\right) \cdot H_{1}\left(\omega_{2}\right)+H_{1}\left(\omega_{2}\right) \cdot H_{1}\left(\omega_{3}\right)\right. \\
\left.+H_{1}\left(\omega_{1}\right) \cdot H_{1}\left(\omega_{3}\right)\right]+G_{d 3} \cdot H_{1}\left(\omega_{1}\right) \cdot H_{1}\left(\omega_{2}\right) \cdot H_{1}\left(\omega_{3}\right) \\
+\frac{1}{3} G_{m d} \cdot\left[H_{2}\left(\omega_{1}, \omega_{2}\right)+H_{2}\left(\omega_{2}, \omega_{3}\right)+H_{2}\left(\omega_{1}, \omega_{3}\right)\right] \\
+\frac{2}{3} G_{d 2} \cdot\left[H_{1}\left(\omega_{1}\right) \cdot H_{2}\left(\omega_{2}, \omega_{3}\right)+H_{1}\left(\omega_{2}\right) \cdot H_{2}\left(\omega_{1}, \omega_{3}\right)\right. \\
\left.\left.+H_{1}\left(\omega_{3}\right) \cdot H_{2}\left(\omega_{1}, \omega_{2}\right)\right]\right\}
\end{gathered}
$$

Although the validity of these transfer functions for large-signal analysis is questionable, they can still be used to qualitatively explain the physical origins of the PA AM/AM and AM/PM distortions.

We consider a two-tone input excitation with amplitudes $\left|V_{g s}\left(\omega_{1}\right)\right|$ and $\left|V_{g s}\left(\omega_{2}\right)\right|$. The time-domain signal corresponds to

$$
x(t)=\operatorname{Re}\left\{\left|V_{g s}\left(\omega_{1}\right)\right| \cdot e^{j \omega_{1} t}+\left|V_{g s}\left(\omega_{2}\right)\right| \cdot e^{j \omega_{2} t}\right\} .
$$

In order to express the input excitation as a cosine carrier modulated, in amplitude, by the purely real $2\left|V_{g s}(\omega)\right| \cdot \cos (\Delta \omega / 2 \cdot t)$ envelope, we need to rewrite eq. (6) as

$$
\begin{aligned}
x(t)=\operatorname{Re}\left\{\left[\left|V_{g s}(\omega)\right| \cdot e^{-j(\Delta \omega / 2) t}\right.\right. & \\
& \left.\left.+\left|V_{g s}(\omega)\right| \cdot e^{j(\Delta \omega / 2) t}\right] \cdot e^{j \omega_{c} t}\right\},
\end{aligned}
$$

where

$$
\begin{gathered}
\omega_{c}=\frac{\omega_{1}+\omega_{2}}{2}, \\
\Delta \omega=\omega_{2}-\omega_{1} .
\end{gathered}
$$

The output time-domain waveform will be given by

$$
\begin{aligned}
y(t)= & \operatorname{Re}\left\{\left|V_{d s}\left(2 \omega_{1}-\omega_{2}\right)\right| \cdot e^{j\left(\left(2 \omega_{1}-\omega_{2}\right) t+\theta_{o 3}\right)}\right. \\
& +\left|V_{d s}\left(\omega_{1}\right)\right| \cdot e^{j\left(\omega_{1} t+\theta_{o 1}\right)}+\left|V_{d s}\left(\omega_{2}\right)\right| \cdot e^{j\left(\omega_{2}+\theta_{o 2}\right)} \\
& \left.+\left|V_{d s}\left(2 \omega_{2}-\omega_{1}\right)\right| \cdot e^{j\left(\left(2 \omega_{2}-\omega_{1}\right) t+\theta_{o 4}\right)}\right\},
\end{aligned}
$$

where $\theta_{o 1}, \theta_{o 2}$ are the fundamental and $\theta_{o 3}, \theta_{o 4}$ the IMD phase variations at the output, and

$$
\begin{aligned}
& V_{d s}\left(2 \omega_{1}-\omega_{2}\right) \\
& \quad=3 H_{3}\left(\omega_{1}, \omega_{1},-\omega_{2}\right) \cdot V_{g s}\left(\omega_{1}\right) \cdot V_{g s}\left(\omega_{1}\right) \cdot V_{g s}\left(\omega_{2}\right)^{*}
\end{aligned}
$$

$$
\begin{gathered}
V_{d s}\left(\omega_{1}\right)=\left[H_{1}\left(\omega_{1}\right)+3 H_{3}\left(\omega_{1}, \omega_{1},-\omega_{1}\right) \cdot\left|V_{g s}\left(\omega_{1}\right)\right|^{2}\right. \\
\left.+6 H_{3}\left(\omega_{1}, \omega_{2},-\omega_{2}\right) \cdot\left|V_{g s}\left(\omega_{2}\right)\right|^{2}\right] \cdot V_{g s}\left(\omega_{1}\right) \quad(12) \\
\begin{aligned}
V_{d s}\left(\omega_{2}\right)= & {\left[H_{1}\left(\omega_{2}\right)+3 H_{3}\left(\omega_{2}, \omega_{2},-\omega_{2}\right) \cdot\left|V_{g s}\left(\omega_{2}\right)\right|^{2}\right.} \\
+ & \left.6 H_{3}\left(\omega_{2}, \omega_{1},-\omega_{1}\right) \cdot\left|V_{g s}\left(\omega_{1}\right)\right|^{2}\right] \cdot V_{g s}\left(\omega_{2}\right)
\end{aligned} \\
V_{d s}\left(2 \omega_{2}-\omega_{1}\right) \\
=3 H_{3}\left(\omega_{2}, \omega_{2},-\omega_{1}\right) \cdot V_{g s}\left(\omega_{2}\right) \cdot V_{g s}\left(\omega_{2}\right) \cdot V_{g s}\left(\omega_{1}\right)^{*} .
\end{gathered}
$$

Therefore, using eqs. (8) and (9), we can rewrite eq. (10) as

$$
\begin{aligned}
y(t)= & \operatorname{Re}\left\{\left[\left|V_{d s}\left(2 \omega_{1}-\omega_{2}\right)\right| \cdot e^{-j\left((3 \Delta \omega / 2) t+\theta_{03}(\omega, \Delta \omega)\right)}\right.\right. \\
& +\left|V_{d s}\left(\omega_{1}\right)\right| \cdot e^{-j\left((\Delta \omega / 2) t+\theta_{01}(\omega, \Delta \omega)\right)} \\
& +\left|V_{d s}\left(\omega_{2}\right)\right| \cdot e^{j\left((\Delta \omega / 2) t+\theta_{02}(\omega, \Delta \omega)\right)}+\mid V_{d s}\left(2 \omega_{2}\right. \\
& \left.\left.\left.-\omega_{2}\right) \mid \cdot e^{j\left((3 \Delta \omega / 2) t+\theta_{04}(\omega, \Delta \omega)\right)}\right] \cdot e^{j \omega_{c} t}\right\} .
\end{aligned}
$$

Contrary to the usual way of identifying AM/AM and $\mathrm{AM} / \mathrm{PM}$ from a time variation of the input and output envelopes, now we have to look for these in eq. (10) via

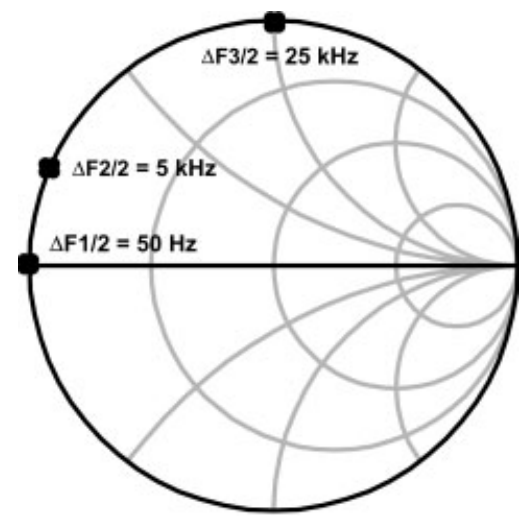

Figure 4. Baseband impedances at three different twotone separation frequencies $\left(\Delta F_{1} / 2, \Delta F_{2} / 2\right.$, and $\left.\Delta F_{3} / 2\right)$. 

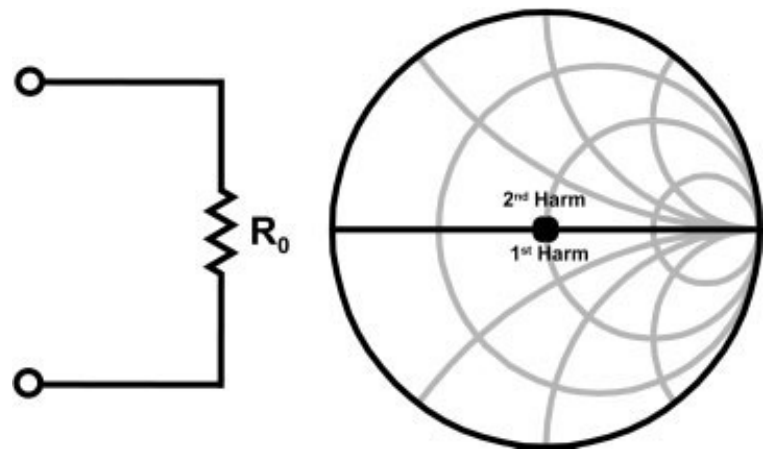

Figure 5. Load $L_{1}$ and its impedances at the frequencies of interest.

their Fourier representation. Amplitude modulation can be described by a real envelope, while phase modulation must involve a complex envelope. So, the presence of the envelope harmonic components at the PA output (the IMD side-bands) describes the envelope amplitude distortion and is thus AM/AM. On the contrary, AM/PM, or output phase modulation, requires an envelope with a non-null imaginary part, or a baseband modulation whose spectrum does not obey the complex conjugate symmetry of pure real signals. So, AM/PM must be identified from the asymmetric amplitudes or phases of the fundamental and IMD components.

As seen from eqs. (8)-(15) and (5), all $\mid V_{d s}\left(2 \omega_{1}-\right.$ $\left.\omega_{2}\right)|,| V_{d s}\left(\omega_{1}\right)|,| V_{d s}\left(\omega_{2}\right)|,| V_{d s}\left(2 \omega_{2}-\omega_{1}\right) \mid$, and $\theta_{o 3}, \theta_{o 1}$, $\theta_{o 2}, \theta_{o 4}$ depend on both $\omega_{c}$ and $\Delta \omega / 2$, which means that, in general, we should expect AM/AM and $\mathrm{AM} / \mathrm{PM}$ variations with the short and long-term dynamics on the amplifier via $\omega_{c}$ and $\Delta \omega / 2$, respectively.

It is these long-term dynamics, shown in eq. (15) by the dependence on $\Delta \omega / 2$, that explain the hysteretic $\mathrm{AM} / \mathrm{AM}$ and $\mathrm{AM} / \mathrm{PM}$ characteristics observed in the studied PA examples.

\section{LOAD-IMPEDANCE IMPACT}

The impact of the load terminations on the AM/AM and AM/PM conversions was studied using a simplified memoryless version of a GaN HEMT [4]. The transistor was biased at class $\mathrm{AB}$ and a nonideal bias-T (Fig. 3) followed by one of four alternative loads was used at the output. Several envelope simulations $[5,6]$ were performed using time-varying envelope stimulus (two-tone signals, centered at $900 \mathrm{MHz}$ ) with different separation frequencies carefully chosen based on knowledge of the
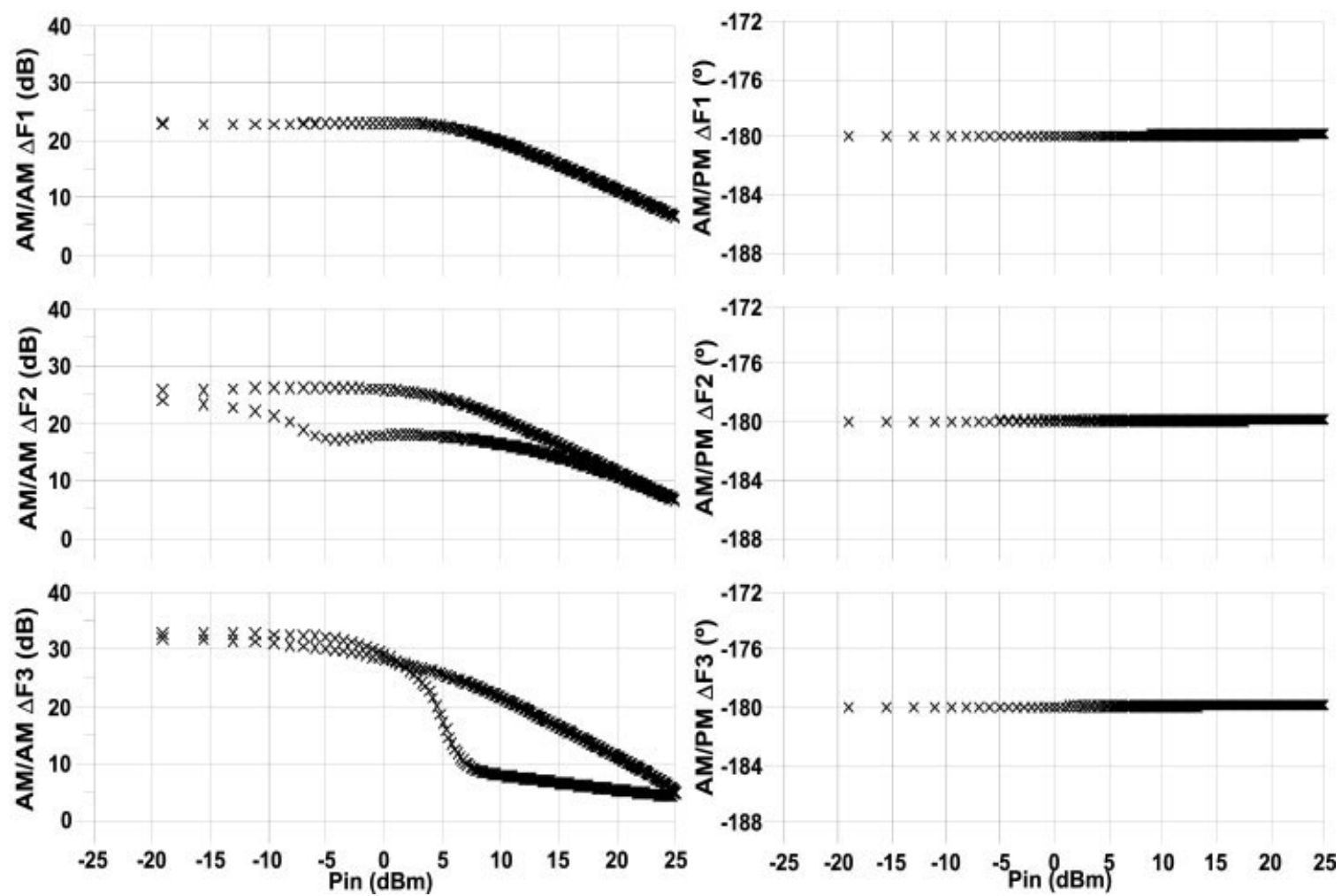

Figure 6. $\mathrm{AM} / \mathrm{AM}$ and $\mathrm{AM} / \mathrm{PM}$ conversions when the active device model is terminated with a nonideal bias- $\mathrm{T}$ and with load $L_{1}$ for three input tone separations $\left(\Delta F_{1}, \Delta F_{2}\right.$, and $\left.\Delta F_{3}\right)$. 

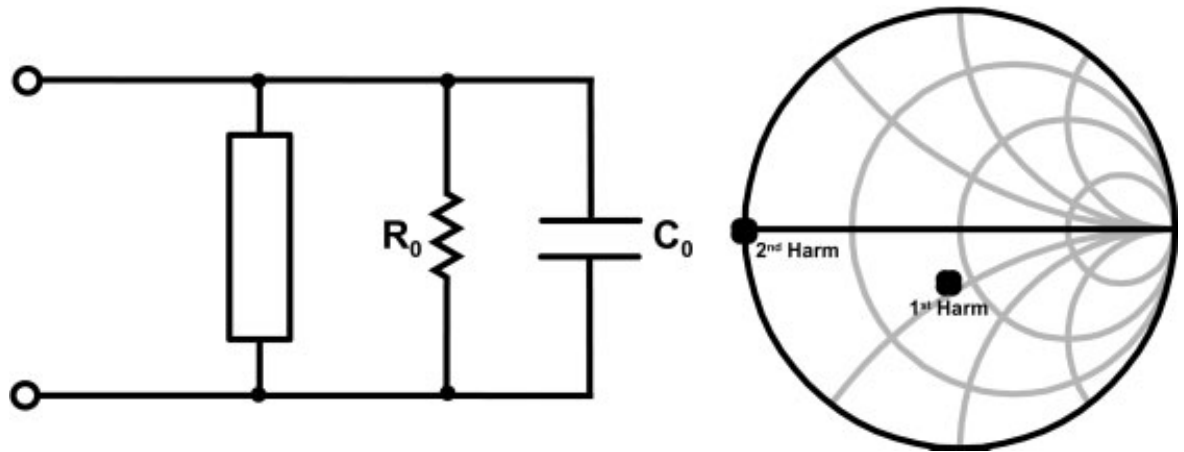

Figure 7. Load $L_{2}$ and its impedances at the frequencies of interest.

PA's output impedance at the baseband components, $\Delta \omega / 2$ (a short circuit, $\Delta F_{1} / 2=50 \mathrm{~Hz}$, or two different reactive terminations, $\Delta F_{2} / 2=5 \mathrm{kHz}$ and $\Delta F_{3} / 2=25$ kHz, see Fig. 4).

Since the quasi-static approximation implies that $i_{D S}$ is a memoryless nonlinearity, it can only present AM/AM conversion. However, the different phase contributions introduced by the device's parasitic reactances and dynamic load impedance $Z_{L}(\omega)$ through the dependence of $i_{D S}$ on $v_{D S}$ will finally establish the overall $\mathrm{PA} \mathrm{AM} / \mathrm{AM}$ and $\mathrm{AM} / \mathrm{PM}$ conversions.
The tests made with tone separation $\Delta F_{1}$ correspond to a static analysis, since the bias- $T$ terminated with the load presents a short circuit to the baseband components. So, in this case, there will be no longterm memory effects visible on the AM/AM or even on the AM/PM conversion plot.

For the other separation frequencies $\left(\Delta F_{2}\right.$ and $\left.\Delta F_{3}\right), \Delta \omega / 2$ long-term dynamics will explain the hysteretic $\mathrm{AM} / \mathrm{AM}$ and $\mathrm{AM} / \mathrm{PM}$ conversions. If that is the case, the PA will not respond instantaneously to its input and the output amplitude and phase will no
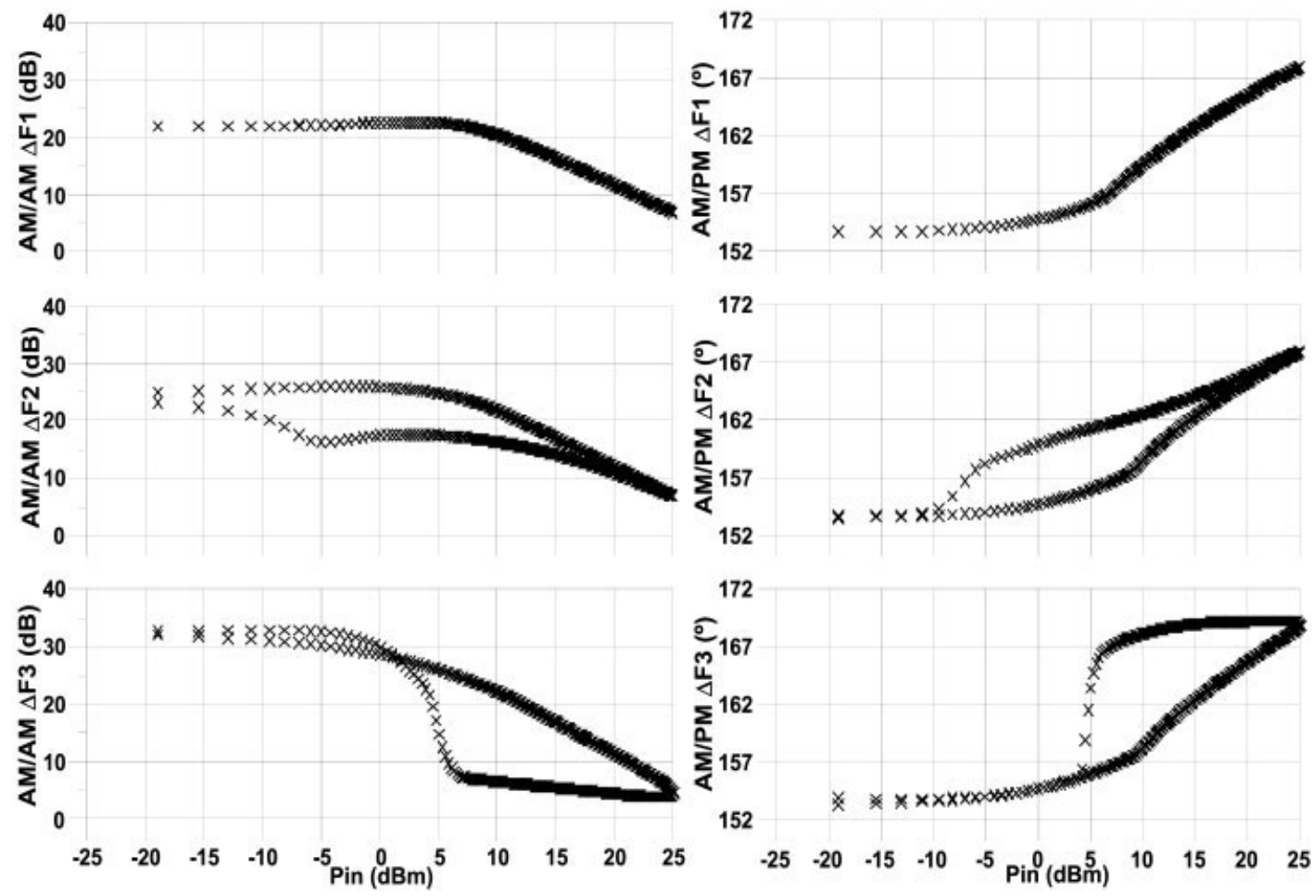

Figure 8. $A M / A M$ and $A M / P M$ conversions when the active device model is terminated with a nonideal bias-T and with load $L_{2}$ for three input tone separations $\left(\Delta F_{1}, \Delta F_{2}\right.$, and $\left.\Delta F_{3}\right)$. 

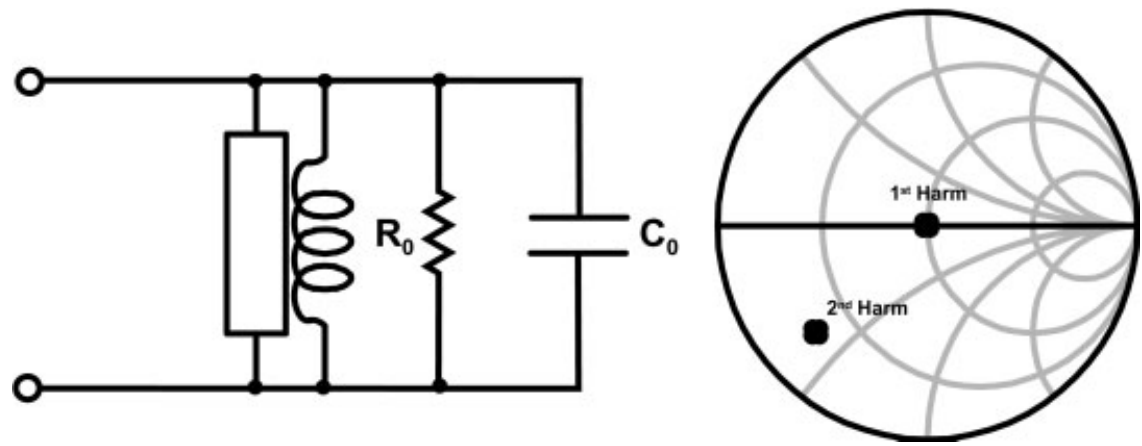

Figure 9. Load $L_{3}$ and its impedances at the frequencies of interest.

longer be single-valued functions of the instantaneous excitation amplitude. They will also depend on the amplifier's state or input history.

\section{A. Active Device Model Terminated with Load $L_{1}$}

The first load (our reference case), load $L_{1}$, is purely resistive. Figure 5 shows the load and its impedance at the frequencies of interest.

Using the band-pass characteristics of our nonlinear model, we can define

$$
\begin{aligned}
\omega_{1} \approx \omega_{2}= & >H_{1}\left(\omega_{1}\right) \approx H_{1}\left(\omega_{2}\right) \approx H_{1}(\omega) \text { and } H_{2}\left(2 \omega_{1}\right) \\
& \approx H_{2}\left(2 \omega_{2}\right) \approx H_{2}\left(\omega_{1}+\omega_{2}\right) \approx H_{2}(2 \omega)
\end{aligned}
$$

Since load $L_{1}$ is purely resistive, from eqs. (3)-(5) and (16) we can see that $H_{1}\left(\omega_{1}\right)=H_{1}\left(\omega_{2}\right), H_{3}\left(\omega_{1}, \omega_{1}\right.$, $\left.-\omega_{1}\right)=H_{3}\left(\omega_{2}, \omega_{2},-\omega_{2}\right)$ are all real values and that $H_{3}\left(\omega_{1}, \omega_{2},-\omega_{2}\right)=H_{3}\left(\omega_{2}, \omega_{1},-\omega_{1}\right) *$ and $H_{3}\left(\omega_{1}, \omega_{1}\right.$, $\left.-\omega_{2}\right)=H_{3}\left(\omega_{2}, \omega_{2},-\omega_{1}\right)^{*}$.

From the previous expressions and from eqs. (11)-(14), it is possible to see that the envelope harmonic components at the PA's output will be
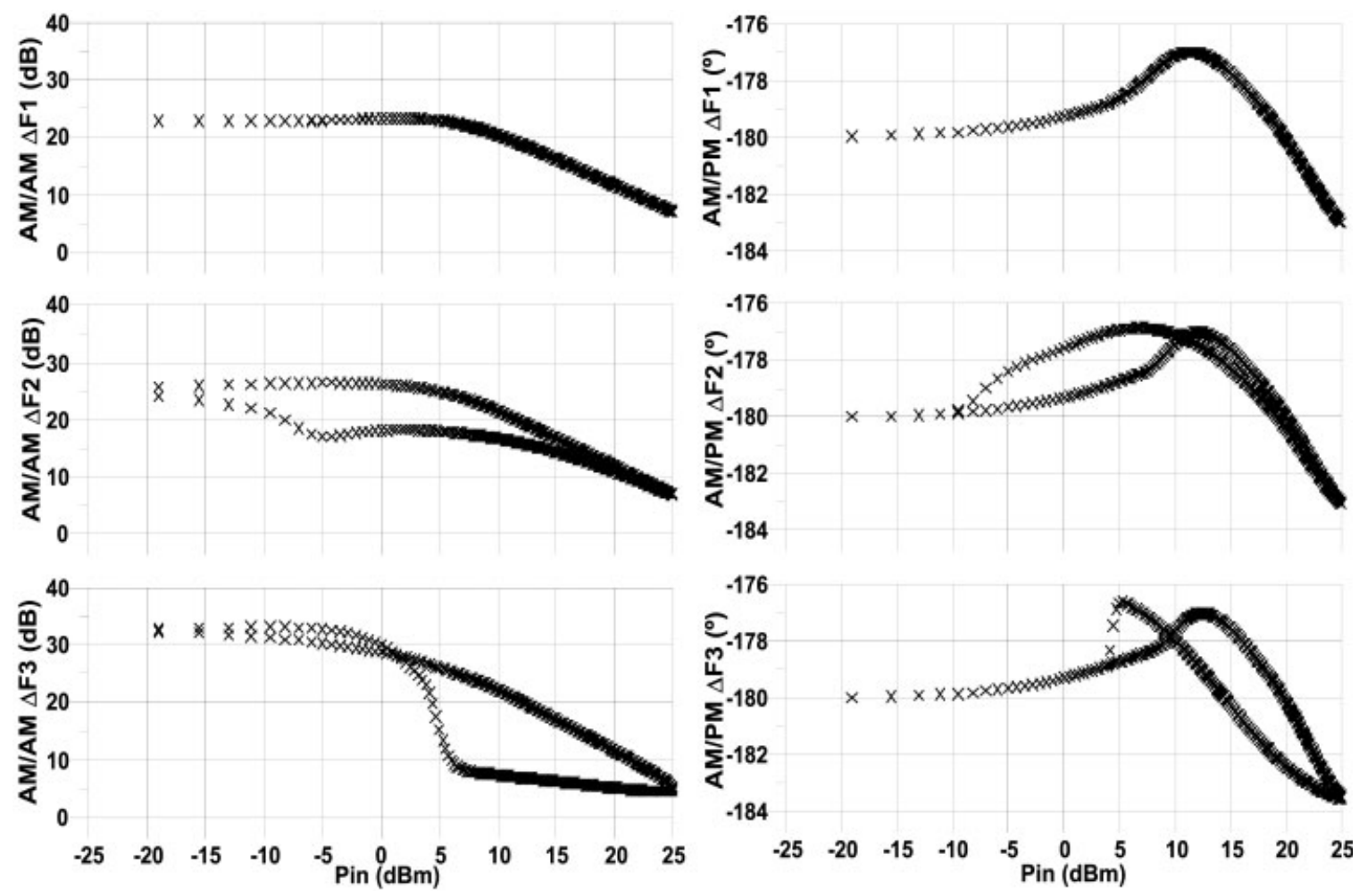

Figure 10. AM/AM and AM/PM conversions when the active device model is terminated with a nonideal bias- $\mathrm{T}$ and with load $L_{3}$ for three input tone separations $\left(\Delta F_{1}, \Delta F_{2}\right.$, and $\left.\Delta F_{3}\right)$. 

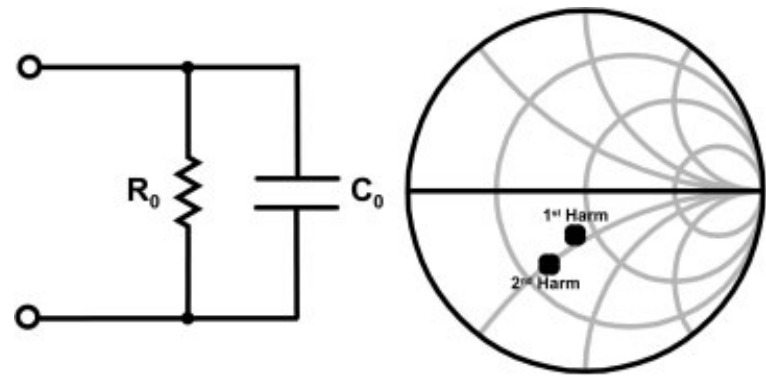

Figure 11. Load $L_{4}$ and its impedances at the frequencies of interest.

nonnull. So, AM/AM will occur. Besides that, as was theoretically explained, since $V_{d s}\left(\omega_{1}\right)=$ $V_{d s}\left(\omega_{2}\right)^{*}$ and $V_{d s}\left(2 \omega_{1}-\omega_{2}\right)=V_{d s}\left(2 \omega_{2}-\omega_{1}\right)^{*}$, no AM/PM conversion will occur. Several envelope simulations of the active device model terminated with load $L_{1}$ for the three different separation frequencies $\left(\Delta F_{1}, \Delta F_{2}\right.$, and $\left.\Delta F_{3}\right)$, were conducted. The AM/AM and AM/PM conversion plots obtained are shown in Figure 6 and the proposed theoretical explanations are fully validated.

\section{B. Active Device Model Terminated with Load $L_{2}$}

The next step was to terminate the active device model with load $L_{2}$, where the resistor is in parallel with a capacitor and stub tuned to short circuit $Z_{L}(2 \omega)$. Figure 7 shows the load and its impedance at the frequencies of interest.

Observing Figure 7, and from eqs. (3)-(5) and (16), it is possible to see that, contrary to the previous case, in spite of $H_{1}\left(\omega_{1}\right)=H_{1}\left(\omega_{2}\right)$ and $H_{3}\left(\omega_{1}, \omega_{1}\right.$, $\left.-\omega_{1}\right)=H_{3}\left(\omega_{2}, \omega_{2},-\omega_{2}\right)$, these are no longer real quantities and $H_{3}\left(\omega_{1}, \omega_{2},-\omega_{2}\right) \neq H_{3}\left(\omega_{2}, \omega_{1},-\omega_{1}\right)^{*}$.

Once again, the envelope harmonic components at the PA's output will be nonnull. So, AM/AM will still occur. Besides that, as it was theoretically explained, since $V_{d s}\left(\omega_{1}\right) \neq V_{d s}\left(\omega_{2}\right)^{*}$ there will also be AM/PM conversion.

Figure 8 shows the AM/AM and AM/PM conversions obtained from several envelope simulations of the active device model terminated with load $L_{2}$ for the three different separation frequencies $\left(\Delta F_{1}, \Delta F_{2}\right.$, and $\Delta F_{3}$ ), where the proposed theoretical explanations are fully validated.
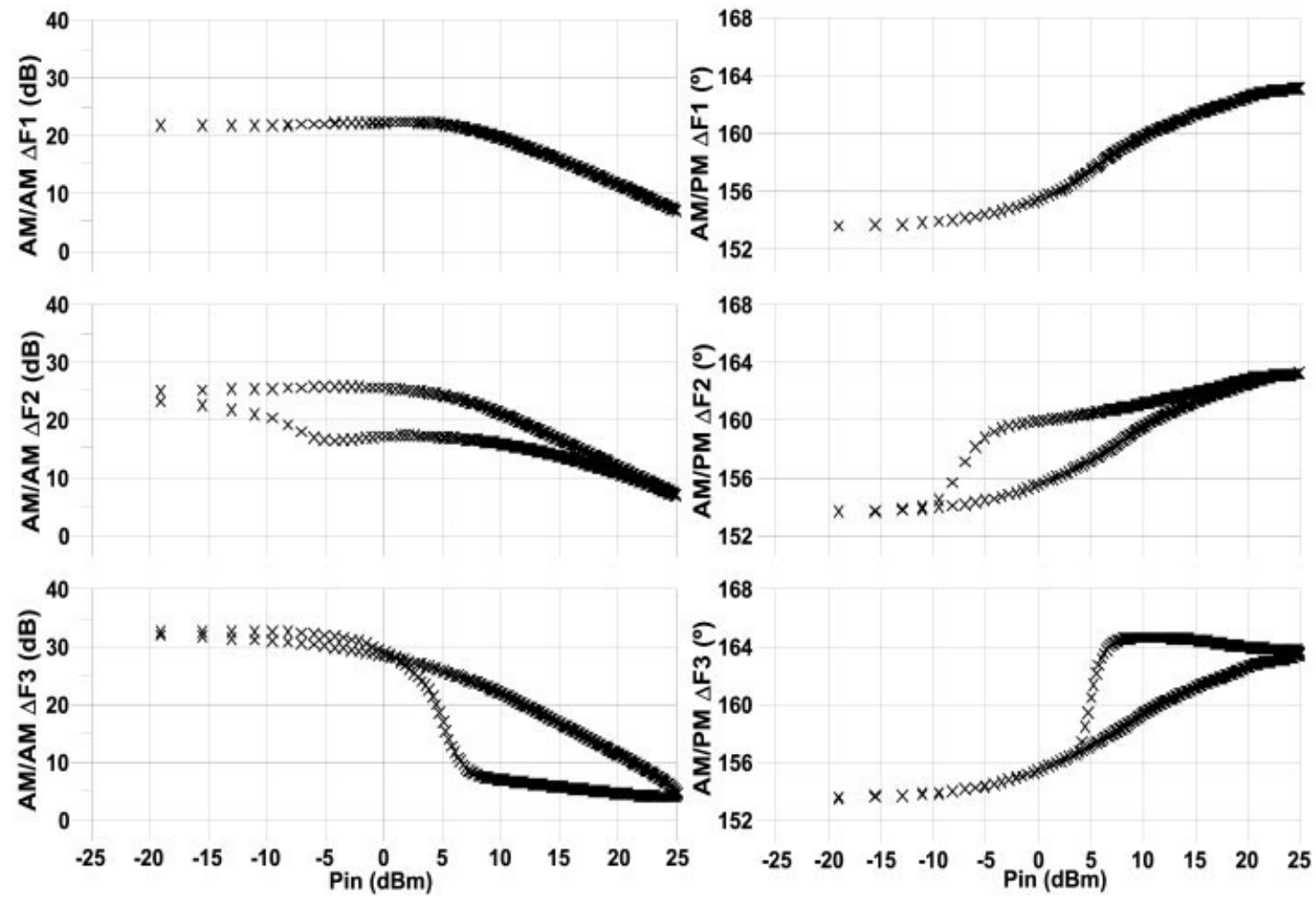

Figure 12. $A M / A M$ and $A M / P M$ conversions when the active device model is terminated with a nonideal bias-T and with load $L_{4}$ for three input tone separations $\left(\Delta F_{1}, \Delta F_{2}\right.$, and $\left.\Delta F_{3}\right)$. 

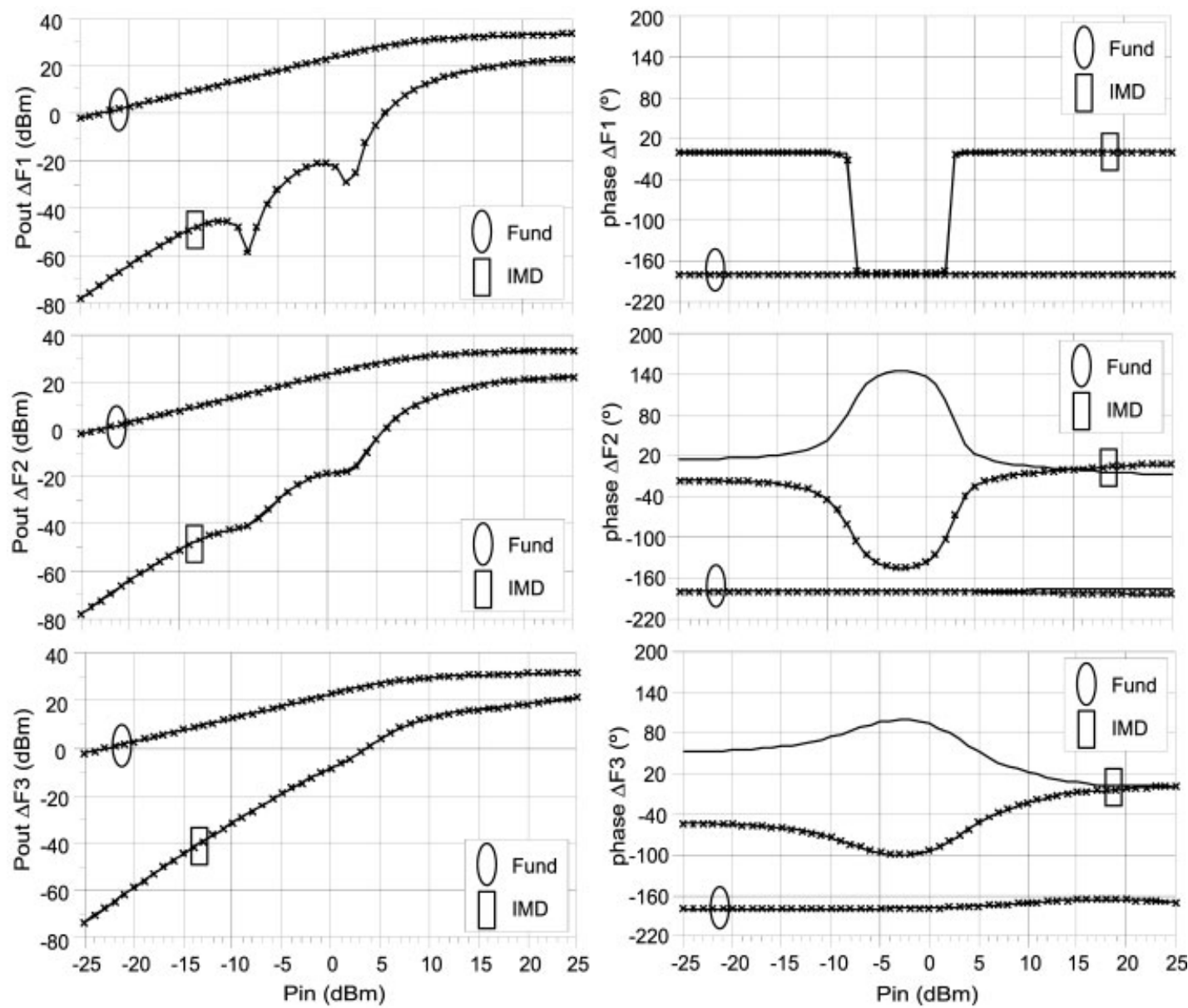

Figure 13. Output power and IMD (amplitude and phase) when the active device model is terminated with a nonideal bias-T and with load $L_{1}$ for three separation frequencies $\left(\Delta F_{1}, \Delta F_{2}\right.$, and $\left.\Delta F_{3}\right)$.

\section{Active Device Model Terminated with Load $L_{3}$}

Next, in order to evaluate the $Z_{L}(2 \omega)$ contribution, a parallel inductance was used to reset the impedance at the fundamental to $50 \Omega$ but leaving a reactive $2^{\text {nd }}$ harmonic termination (load $L_{3}$ ). Figure 9 shows the load and its impedance at the frequencies of interest.

Once again, from eqs. (3)-(5) and (16), it is possible to see that $H_{1}\left(\omega_{1}\right)=H_{1}\left(\omega_{2}\right)$ are real values. On the contrary, $H_{3}\left(\omega_{1}, \omega_{1},-\omega_{1}\right)=H_{3}\left(\omega_{2}, \omega_{2}\right.$, $\left.-\omega_{2}\right)$ are not real quantities. In addition, the dependence on $2 \omega$ implies that $H_{3}\left(\omega_{1}, \omega_{2},-\omega_{2}\right) \neq H_{3}\left(\omega_{2}\right.$, $\left.\omega_{1},-\omega_{1}\right)^{*}$.

For the reasons explained above, this PA circuit will manifest AM/AM and, since $V_{d s}\left(\omega_{1}\right) \neq V_{d s}\left(\omega_{2}\right)^{*}$, there will also be AM/PM conversion.

Figure 10 shows the AM/AM and AM/PM conversions obtained from several envelope simulations of the active device model terminated with load $L_{3}$ for the three different separation frequencies $\left(\Delta F_{1}, \Delta F_{2}\right.$, and $\Delta F_{3}$ ).

\section{Active Device Model Terminated with Load $L_{4}$}

Finally, we loaded the active device with load $L_{4}$. This is only a resistor in parallel with a capacitor which provides a reactive termination to both the fundamental and $2^{\text {nd }}$ harmonics. Figure 11 shows the load and its impedance at the frequencies of interest.

Figure 12 shows the AM/AM and AM/PM conversions obtained from several envelope simulations of the active device terminated with load $L_{4}$ for the three different separation frequencies $\left(\Delta F_{1}, \Delta F_{2}\right.$, and $\left.\Delta F_{3}\right)$. As expected from the previous analysis, since this case is the aggregate of the last two, once again the $\mathrm{AM} / \mathrm{AM}$ and AM/PM conversions will occur. 

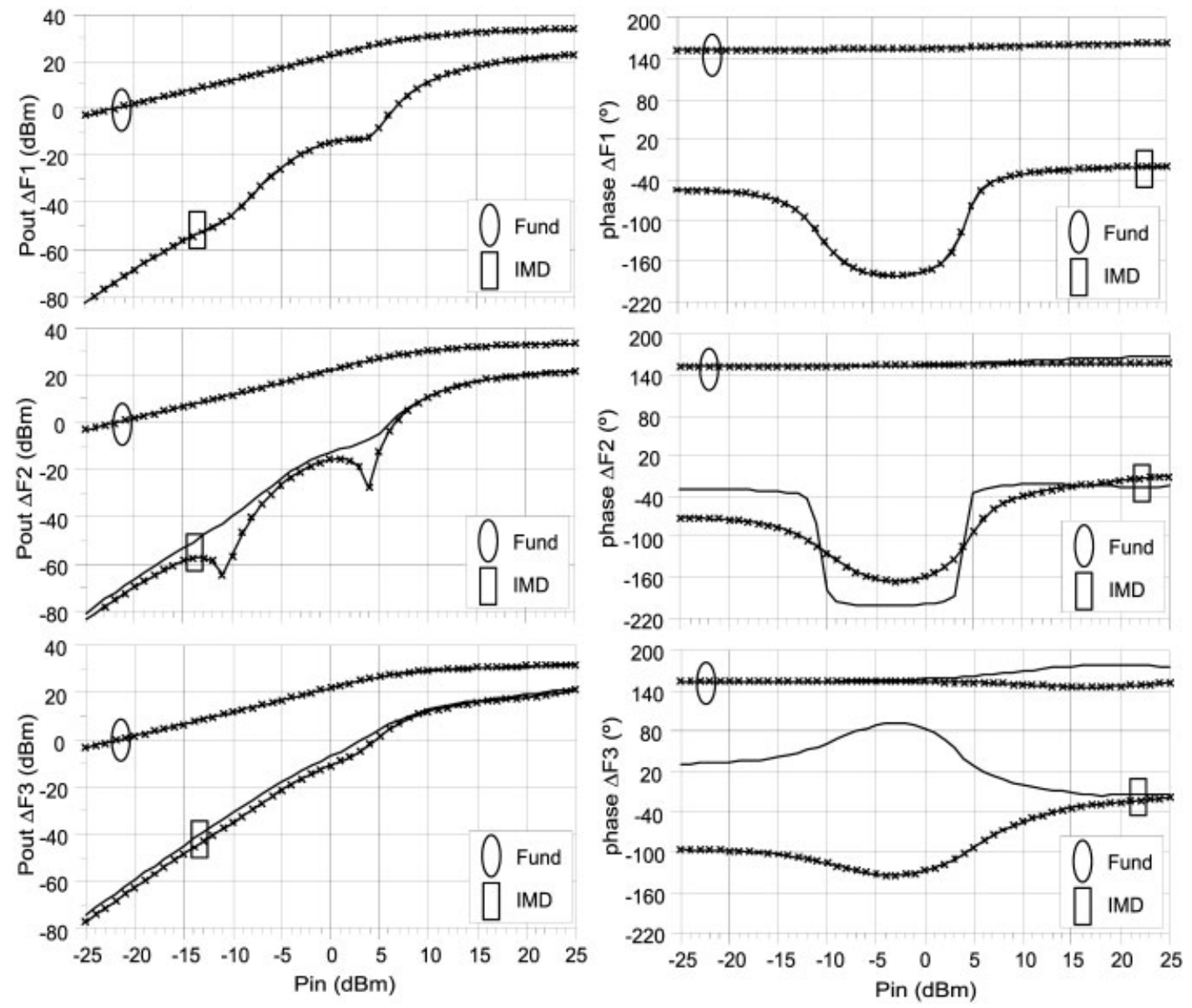

Figure 14. Output power and IMD (amplitude and phase) when the active device model is terminated with a nonideal bias-T and with load $L_{4}$ for three input tone frequency separations $\left(\Delta F_{1}\right.$, $\Delta F_{2}$, and $\left.\Delta F_{3}\right)$.

\section{MEMORY-EFFECTS MANIFESTATIONS ON THE TWO-TONE IMD TESTS}

After the study of the AM/AM and AM/PM conversions' behavior with load terminations, the next stage was to recognize patterns that can lead to the identification of memory effects on the two-tone IMD distortion tests, for a certain PA circuit.

As is known, one common manifestation of long-term memory is the amplitude and phase variation of the IMD side-bands with separation frequency. Thus, several two-tone harmonic balance simulations were conducted with the active device terminated with two of the four loads previously studied (loads $L_{1}$ and $L_{4}$ ).

Figure 13 shows the fundamental and IMD amplitude and phase measured at the output of the active device when terminated with load $L_{1}$ for three distinct two-tone excitations of $\Delta F_{1}, \Delta F_{2}$, and $\Delta F_{3}$ separation frequencies.

The evolution of the IMD pattern from $\Delta F_{1}$ to $\Delta F_{3}$ is the sought-for symptom of long-term memory. This exclusively originated in the reactive baseband termination manifests itself across the variation from one to another frequency separation. Indeed, because the circuit is resistive to any other frequency component except $\Delta \omega$, the IMD side-bands (at $2 \omega_{2}-\omega_{1}=\omega_{2}+$ $\Delta \omega$ and $\left.2 \omega_{1}-\omega_{2}=\omega_{1}-\Delta \omega\right)$ show a symmetric phase behavior.

Substituting load $L_{1}$ for load $L_{4}$ and resimulating the whole circuit in the same conditions, we obtained the plots presented in Figure 14.

In this case, besides the amplitude and phase variations of the IMD distortion with separation frequency, we can also see another well-documented long-term memory manifestation: IMD asymmetry. As shown in [7], this is due to the presence of a 
simultaneous reactive termination at both the baseband and the $2^{\text {nd }}$ harmonic.

\section{CONCLUSION}

A comprehensive study of the different in-band and out-of-band load terminations' impact on the AM/AM and AM/PM conversions has been performed theoretically, based on an approximate Volterra series analysis, and validated through envelope simulations.

Four different loads were considered and several two-tone input signals, with different separation frequencies, were also used. This allowed, on one hand, the isolation of the fundamental and $2^{\text {nd }}$-harmonic contributions for the overall AM/AM and AM/PM conversions and, on the other hand, enabled a study of the long-term memory effects that arise from the presence of reactive based-band terminations.

The theoretical dynamic-envelope analysis enabled a fast and intuitive way of determining whether a certain PA circuit can present memory effects when dealing with certain input signals with time-varying envelopes.

For the sake of completeness, this study was then complemented by the more common memory-effects observation gathered from swept frequency-separation two-tone IMD tests.

We believe this study will be of help to PA designers for understanding and preventing amplitude- and phasesignal impairments, since it presents a theoretical explanation for both conversions and shows that the harmonic and baseband terminations are crucial for modelling AM/AM, AM/PM, and two-tone IMD.

\section{ACKNOWLEDGMENTS}

The authors would like to thank Portuguese Science and Technology Foundation, F.C.T., for the Ph.D. grant (ref.
$11323 / 2002)$, given to the first author, and financial support provided under Project POCTI/ESE/45050/2002 MEGaN.

The research reported here was also performed in the context of the network TARGET_- "Top Amplifier Research Groups in a European Team" and supported by the Information Society Technologies Programme of the EU under contract IST-1-507893-NOE (www.target-net.org).

\section{REFERENCES}

1. J.C. Pedro and N.B. Carvalho, Intermodulation distortion in microwave and wireless circuits, Artech House, Norwood, MA, 2003.

2. A.A. Moulthrop, C.J. Clark, C.P. Silva, and M.S. Muha, A dynamic AM/AM and AM/PM measurement technique, IEEE MTT-S Int Microwave Symp Dig 3 (1997), 1455-1458.

3. J.C. Pedro, N.B. Carvalho, and P.M. Lavrador, Modeling nonlinear behavior of band-pass memoryless and dynamic systems, IEEE MTT-S Int Microwave Symp Dig 3 (2003), 2133-2136.

4. P.M. Cabral, J.C. Pedro, and N.B. Carvalho, New nonlinear device model for microwave power $\mathrm{GaN}$ HEMTs, IEEE MTT-S Int Microwave Symp Dig 1 (2004), 51-54.

5. D. Sharrit, New method of analysis of communication systems, MTT-S Nonlinear CAD Wkshp, San Francisco, CA, 1996.

6. E. Ngoya and R. Larchevèque, Envelope transient analysis: A new method for the transient and steady-state analysis of microwave communications circuits and systems, IEEE Microwave Theory Tech Symp Dig 3 (1996), 1365-1368.

7. N.B. Carvalho and J.C. Pedro, A comprehensive explanation of distortion sideband asymmetries, IEEE Trans Microwave Theory Tech 50 (2002), 2090-2101.

\section{BIOGRAPHIES}

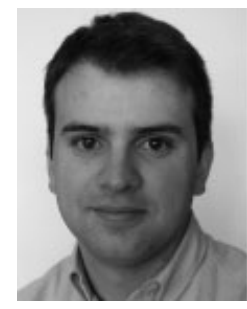

Pedro M. Cabral was born in Portugal in October 1979. He received his degree in electrical engineering in 2002 from University of Aveiro, Portugal. Since then, he has been pursuing a Ph.D. degree in nonlinear transistor modeling and lecturing several Lab classes at the same university. In 2002, he received the prize for the Best Electrical Engineering Student at the University of Aveiro and in 2004 he was finalist of the Student Paper Competition presented at the IEEE International Microwave Symposium. His main interests are in nonlinear modeling and design of microwave circuits and active devices.

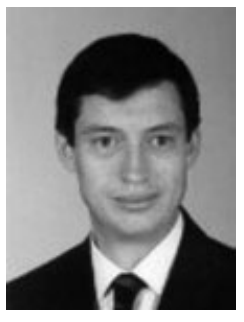

José C. Pedro was born in Espinho, Portugal on March 7, 1962. He received diploma, doctoral, and habilitation degrees in electronics and telecommunications engineering, from University of Aveiro, Portugal, in 1985, 1993, and 2002, respectively. From 1985 to 1993, he was an Assistant Lecturer at University of Aveiro, and he has been a Professor since 1993. Currently he is a Professor at the same university, and a Senior Research Scientist at the Institute of Telecommunications. His main scientific interests include active device modeling and the analysis and design of various 
nonlinear microwave and optoelectronics circuits, in particular, the design of highly linear multicarrier power amplifiers and mixers. $\mathrm{He}$ is the lead author of "Intermodulation Distortion in Microwave and Wireless Circuits" (Artech House, 2003), has authored or coauthored more than 100 papers in international journals and symposia, and serves as a reviewer for various publications and scientific events, such as the IEEE MTT Transactions, International Journal of RF and Microwave Computer-Aided Engineering, and MTT-IMS. Currently, he is an Associate Editor of IEEE MTT Transactions. He received the Marconi Young Scientist Award in 1993 and the 2000 Institution of Electrical Engineers (IEE) Measurement Prize.

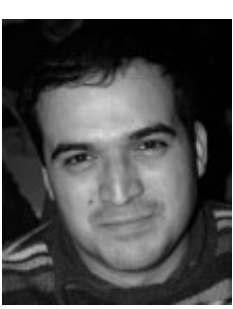

Nuno B. Carvalho was born in Luanda in 1972. He received diploma and doctoral degrees in electronics and telecommunications engineering from the University of Aveiro, Aveiro, Portugal in 1995 and 2000, respectively. From 1997 to 2000, he was an Assistant Lecturer at the same university and he has been a Professor since 2000. Currently he is an Associate Professor at the same University, and a Senior Research Scientist at the Institute of Telecommunications. He has worked as a Scientist Researcher at the Telecommunications Institute, and was engaged in different projects on nonlinear $\mathrm{CAD}$, and circuits and RF systems integration. His main research interests include CAD for nonlinear circuits, design of highly linear RF-microwave power amplifiers, and measurement of nonlinear circuits/systems. He is a member of the Portuguese Engineering Association and a Senior Member of IEEE. He was the recipient of the 1995 University of Aveiro and Portuguese Engineering Association prize for the Best Student at the University of Aveiro, the 1998 Student Paper Competition (third place) presented at the IEEE International Microwave Symposium, and the 2000 IEE Measurement Prize. He has been a reviewer for several magazines and is a member of the IEEE Transactions on Microwave Theory and Techniques Review Board. He is coauthor of the book "Intermodulation in Microwave and Wireless Circuits" (Artech House, 2003). 